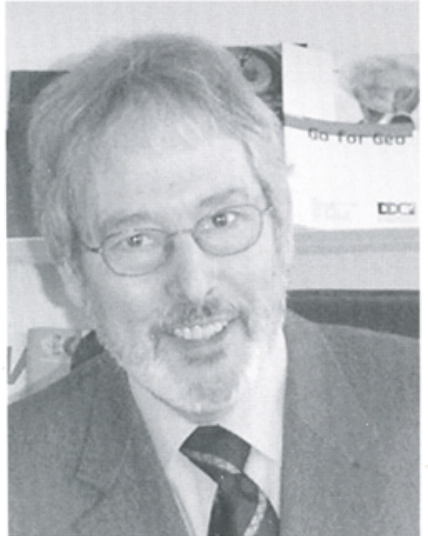

Rolf Harbeck

\section{Kartographie - souveräner Leistungsbaustein im Geoinformationswesen}

Liebe Leserin, lieber Leser, der diesjährige Kartographentag mit Staatsgrenzen übergreifenden Problemthemen und der immer wiederkehrenden Frage nach der Stellung unseres Faches in den Geowissenschaften liegt hinter uns. Haben wir Antworten erhalten? Wir nehmen wahr, dass die professionelle kartographische Darstellung als modernes Multimedium der Information und Kommunikation viele Menschen zunehmend interessiert und herausfordert: Wissenschaftler und Praktiker, Softwaredesigner und Unternehmer, Anwender und Konsumenten und - die Politiker. Wir selbst schätzen die eigenständige Kartographie als Kopfleistung, als kreatives Gestalten und als Handwerk. Wir sehen, dass Kartographie bedeutet, raumbezogene Informationen über die reale Welt den Menschen zu vermitteln: anschaubar, hörbar und greifbar. Dass hinter dieser Aufgabe heute oft eine Geodatenbank steht, ist unsere große Chance. Denn dieses Datenpotenzial müssen wir erschließen, können wir vielfältig einsetzen und müssen wir verständlich machen. Damit wird Kartographie zum souveränen Leistungsbaustein im großen Gebäude des Geoinformationswesens.

Das moderne Themenspektrum der Kartographie ist breit gefächert. Klassische Fundamentalthemen wie Zeichenwahrnehmung, Kartengestaltung und Generalisierung gehören dazu, Forschungs- und Entwicklungsaufgaben also, für die durchgreifende rechnergestützte Lösungen näher rücken. Aber auch Fragen zur dynamischen und mobilen Kartographie, zu Funktionalität und Leistungsfähigkeit von Softwarebausteinen oder zum Umgang mit Internetplattformen wollen behandelt und im Erfahrungsaustausch einer Beantwortung näher gebracht werden. Konzepte und Strategien der Interessenverbände und Politiker berühren die Kartographie und fordern sie zur Stellungnahme heraus. Fragen berufs- ständischen Denkens und Handelns, wirtschaftlichen Arbeitens und Produzierens bleiben dabei nicht ausgeklammert. Und eine lebhafte Thematik begleitet uns ständig: Wie erleben wir Kartographie am Arbeitsplatz, in der Gesellschaft, in unserem Verband?

Unsere Kartographischen Nachrichten sind für dieses Themenspektrum offen, das in seiner Breite auch eine Herausforderung für die Schriffleitung und das editorial board bedeutet. Dass Sie, liebe Leserinnen und Leser, repräsentiert durch die Mitgliederversammlung der DGFK am 29. Mai 2003 in Bad Krozingen, mir das Vertrauen ausgesprochen haben, an dieser Herausforderung maßgeblich mitzuwirken, dafür möchte ich Ihnen an dieser Stelle herzlich danken.

Mit der August-Ausgabe der KN halten Sie ein Heft in Händen, das Ausdruck dieser Themenvielfalt ist und sich auch an die Teilnehmerinnen und Teilnehmer der INTERGEO 2003 vom 17. bis 19. September in Hamburg wendet. Im Aufsatzteil setzt sich Norbert Hackner aus der Sicht eines kartographischen Verlages mit den veränderten Produktionsbedingungen in der Kartographie auseinander.

Sven Burdack, Nicole Ueberschär und Jürgen Schweikart vergleichen am Beispiel interessanter geothematischer Anwendungen die Internetformate Flash und SVG. Für die Stadt Dresden zeigen Grit Steinbach, Manfred Buchroithner und Hans Stieber auf, wie die Daten des Straßenknotennetzes durch Überführung in ein ATKIS-konformes Datenmodell direkt für die Herstellung einer Stadtübersichtskarte genutzt werden können. Und neben vielen Berichten aus Forschung, Praxis und dem Kollegenkreis machen Sie neue Bekanntschaft mit einem "guten, alten" Produkt der Kartographie: dem Globus.

Ich wünsche Ihnen Freude und Genuss beim Blättern, Lesen und Studieren.

\section{Tor Aaccuch}

